\title{
Random Energy Model at Complex Temperatures.
}

\author{
D.B. Saakian \\ Yerevan Physics Institute, Alikhanian Brothers St. 2, Yerevan 375036, Armenia \\ and \\ Universidad de La Frontera, Departamento de Cienas Fisicas, Casilla 54-D, Temuco, Chile.
}

\begin{abstract}
The complete phase diagram of Random Energy Model (REM) is obtained for complex temperatures using the method proposed by Derrida. We find the density of zeroes for statistical sum. Then the method is applied to Generalized Random Energy Model (GREM). This allows us to propose new analytical method for investigating zeroes of statistical sum for finite-dimensional systems.
\end{abstract}

Random Energy Model (REM) [1] is one of the most popular contemporary models of statistical mechanics. Besides its direct applications in spin-glasses the model has a wide range of employments in very different areas of the modern theoretical physics and biophysics. Recently, several new, and more intimate applications have been founded in the theories of well-developed turbulence [2], and strings [3-5]. On the other hand, it is well-known that the model allows to consider the fundamental problems of information theory in the language of statistical mechanics. In particular, problems connected with transmission of information through a noisy channel can be formulated and effectively solved in the language of REM [6,7]. This astonishing range of different applications cannot be accidental one: It seems, that REM is a paradigm for a complex system physics, or at least contains all essential ingredients of that physics. In other words, the nature sings his sacral songs using REM's language.

The thermodynamical structure of REM has simple structure but at the same contains all essential ingradients of phase transitions [1, 11, 12]: There is well defined critical temperature $T_{c}$ which can be obtained from clear and semi-intuitive physical reasons. For $T<T_{c}$ the system is frozen in the spin-glass phase with one level of replica symmetry breaking. Besides this rough thermodynamical phase transition there are some "mild", mesoscopic phase transitions in the domain $\left(\tilde{T}, T_{c}\right), \tilde{T}>T_{c}$ [1, 12. The temperature $\tilde{T}$ strongly depends from the concrete form of energy's distribution; it can be infinite for the gaussian distribution [1] but it has some finite value for REM generated by more physical dilute hamiltonians [12]. This rich and interesting spectra of phase transitions waits its complete and exhaustive investigation, and we hope that the present work will shed some light to this question also.

It is well-known that structure and properties of phase transitions can be investigated through analytical properties of thermodynamical quantities in the complex plane of temperature or/and magnetic field 18] 17 [16]. Indeed, if a phase transition is assosiated with singular behavior of thermodynamic potential in the thermodynamic limit (in our case it is free energy or statistical sum), then by consideration of its analytical properties in the complex plane the important physical information can be obtained. The method was proposed by Yang and Lee [14] (for the case of complex magnetic fields), and Fisher [15] (for the case of complex temperatures). It has large variety of different applications in statistical physics; furthermore it is one one of the most exact and powerful methods for estimating critical indices and constructing phase diagrams. In particular, the method is applied for investigation of phase transitions in disordered or strongly frustrated systems because sometimes all other methods are not very informative or hard for realization. Resently with help of this method a dense domain of phase transitions has been obtained in a non-disordered but fully-frustrated model [16].

REM has been investigated in complex field/temperature plane $[8,10]$. The goal of the present paper is to continue this program, and to derive the complete phase diagram. Furthermore, we propose a new method for investigating analytical properties of thermodynamical quantities of finite-dimensional systems. There is another, perhaps more fundamental motivation for the present investigation: it was obtained resently that REM at complex temperatures is closely connected with strings [4] [5].

In disordered systems there are averaging with the canonical Gibbs distribution at given temperature, and averaging by frozen disorder. In REM and related models energy levels are considered as random quantities, and the distribution is derived from a concrete "microscopic" Hamiltonian [1] [2].

$$
H=-\sum_{1 \leq i_{1}<i_{2} . .<i_{p} \leq N} j_{i_{1} . . i_{p}} s_{i_{1}} . . s_{i_{p}}
$$

Here $s_{i}$ are \pm 1 spins, $j_{i_{1} . i_{p}}$ are quenched couplings with normal distribution and a proper scale

$$
\rho\left(j_{i_{1} . . i_{p}}\right)=\sqrt{\frac{2 C_{p}^{N}}{N \pi}} \exp -2 C_{p}^{N} j_{i_{1} . . i_{p}}^{2} / N
$$


where $C_{p}^{N}=N ! / p !(N-p)$ !. It is possible to prove, that in the limit of large $p$ all energy levels are distributed independently with probability

$$
P(E)=\sqrt{\frac{1}{N \pi}} \exp \left(-E^{2} / N\right)
$$

Let us define partition function and free energy. Consideration of complex temperatures is some tool for investigating analitical properties of thermodynamical quantities. In particular, the usual definitions of statistical physics should be adapted to this purpose. Partition function is defined as usually:

$$
Z=\sum_{\alpha} e^{-\beta E_{\alpha}}
$$

Further, at the thermodynamical limit different expressions like

$$
<\ln |Z|>,<\ln |\operatorname{Re} Z|>
$$

are identical. In a slightly cavalier fashion we will refer to the quantity $\langle\ln |\operatorname{Re} Z|\rangle$ as free energy, taking into account that for the case of real temperatures it is related simply to the usual free energy.

$$
\langle\ln |\operatorname{Re} Z|\rangle=\int_{0}^{\infty} d t \frac{e^{-t}}{t}-\left\langle\int_{0}^{\infty} d t \frac{e^{-t|\operatorname{Re} Z|}}{t}\right\rangle=\int_{0}^{\infty} d t \ln t e^{-t}+\int_{0}^{\infty} \ln t d\left\langle e^{-t|\operatorname{Re} Z|}\right\rangle
$$

where $\lambda=\beta \sqrt{N}$, and $\langle\ldots\rangle$ means averaging by the normal distribution. Further,

$$
\left\langle e^{-t|\operatorname{Re} Z|}\right\rangle=\left\langle e^{-\phi}\right\rangle=\frac{1}{\sqrt{\pi}} \int_{-\infty}^{\infty} d z e^{-z^{2} / 4} f(z \sqrt{t}, \lambda)^{2^{N}}
$$

and

$$
f(A, \lambda)=\frac{1}{\sqrt{\pi}} \int_{-\infty}^{\infty} d x e^{-x^{2}+i \frac{A}{2}(\exp (\lambda x)+\exp \bar{\lambda} x)}
$$

here we have $\beta \equiv \beta_{1}+\beta_{2}, \lambda \equiv \lambda_{1}+i \lambda_{2}=\sqrt{N} \beta$

We see that $|f(A, \lambda)|$ is always less than 1 . When $\ln A \gg 0$ the $f(A, \lambda)$ is exponentially suppressed and we get $f(A, \lambda) \approx 1$, while $\ln A \ll U$. To find asymptotics it is convenient to use another representation, like [8]

$$
\begin{gathered}
f(A, \lambda)=\frac{1}{2 \pi} \int_{-i \infty}^{i \infty} d x e^{\frac{\lambda_{1}^{2} x^{2}}{4}} \int_{-\infty}^{\infty} d y e^{\lambda_{1} x y-\frac{i A}{2}\left(e^{\lambda y}+e^{\bar{\lambda} y}\right)}= \\
=\frac{1}{2 \pi} \int_{-i \infty}^{i \infty} d x e^{\frac{\lambda_{1}^{2} x^{2}}{4}} \int_{0}^{\infty} d y y^{x-1} e^{\frac{i A}{2}\left(y^{1+i \lambda_{2} / \lambda_{1}}+y^{1-i \lambda_{2} / \lambda_{1}}\right)}
\end{gathered}
$$

In the formula (4) the integral is taken along the line, which passes the point $x=0$ on the right side. To calculate asymptotic we should move the integration line at left to catch the saddle point. There is a simple pole at point 0 . It is easy to check,that the corresponding residue equals 1.

$$
f(A, \lambda)=1+\int_{-i \infty+\varepsilon}^{i \infty-\varepsilon} d x e^{\frac{\lambda_{1}^{2} x^{2}}{4}-x \ln A} g(x, A)
$$

where

$$
g(x, A)=\int_{0}^{\infty} d y y^{x-1} e^{i y \cos \lambda_{2} / \lambda_{1} \ln y / A}
$$

This representation is useful, when the saddle point $x$ belongs to the interval $(0,-1)$. Function $g(x, A)$ has a complicated singularity at point -1 . We need in asymptotic at $\lambda \sim \sqrt{N}, \ln A \sim N$ :

$$
f(A, \lambda)-1 \approx \exp \left[-\frac{\ln A^{2}}{\lambda_{1}^{2}}\right]
$$


where we missed pre-exponents.

There are another 2 modes for $f(A, \lambda)$, besides (7). In order to calculate them we are considering derivatives:

$$
\begin{gathered}
\frac{d}{d A} f(A, \lambda)=\frac{i}{4 \sqrt{\pi}} \int_{-i \infty}^{i \infty} d x e^{-x^{2}+\frac{i A}{2}\left(e^{\lambda x}+e^{\bar{\lambda} x}\right)}\left(e^{\lambda x}+e^{\bar{\lambda} x}\right)= \\
\frac{i}{4 \sqrt{\pi}} e^{\frac{\lambda^{2}}{4}} \int_{-i \infty}^{i \infty} d x e^{-x^{2}+\frac{i A}{2}\left(e^{\frac{\lambda^{2}}{4}+\lambda x}+e^{\frac{|\lambda|^{2}}{4}+\bar{\lambda} x}\right)+} \\
+\frac{i}{4 \sqrt{\pi}} e^{\frac{\lambda^{2}}{4}} \int_{-i \infty}^{i \infty} d x e^{-x^{2}+\frac{i A}{2}\left(e^{\frac{|\lambda|^{2}}{4}+\lambda x}+e^{\frac{\bar{\lambda}^{2}}{4}+\bar{\lambda} x}\right)}= \\
=\frac{i e^{\frac{\lambda^{2}}{4}}}{4 \pi} \int_{-i \infty}^{i \infty} d x e^{\frac{\lambda_{1}^{2} x^{2}}{4}-\left(\ln A+\frac{|\lambda|^{2}}{4}\right) x} g(x, A)+\frac{i e^{\frac{\lambda^{2}}{4}}}{4 \pi} \int_{-i \infty}^{i \infty} d x e^{\frac{\lambda_{1}^{2} x^{2}}{4}-\left(\ln A+\frac{|\lambda|^{2}}{4}\right) x} g(x, A)
\end{gathered}
$$

Eventually we have (after shift of integration line)

$$
\begin{gathered}
f_{A}^{\prime} \approx \frac{i\left(e^{\lambda^{2} / 4}+e^{\bar{\lambda}^{2} / 4}\right)}{2} \\
f_{A} \approx 1+i A\left(e^{\lambda^{2} / 4}+e^{\left.\bar{\lambda}^{2} / 4\right)} \approx 1+i A e^{\left(\lambda_{1}^{2}-\lambda_{2}^{2}\right) / 4}\right.
\end{gathered}
$$

The similar approach gives

$$
\begin{gathered}
f_{A}^{\prime \prime} \approx-\frac{e^{\lambda_{1}^{2}}}{4 \pi} \int_{-i \infty}^{i \infty} d x e^{\frac{\lambda_{1}^{2} x^{2}}{4}-\left(\ln A+\frac{|\lambda|^{2}}{4}\right) x} g(x, A) \\
f(A, \lambda) \approx 1-\frac{A^{2} e^{\lambda_{1}^{2}}}{4}
\end{gathered}
$$

So we have three phases

$$
\begin{gathered}
e^{-\phi}=\frac{1}{\sqrt{2 \pi}} \int_{-i \infty}^{i \infty} d z \exp \left(-z^{2} / 4+2^{N} z t e^{\frac{\lambda_{1}^{2}-\lambda_{2}^{2}}{4}}\right)=\exp \left(-t^{2} 4^{N} e^{\frac{\lambda_{1}^{2}-\lambda_{2}^{2}}{4}}\right) \\
e^{-\phi}=\frac{1}{\sqrt{\pi}} \int_{-i \infty}^{1 \infty} d z \exp \left[-z^{2} / 4-\frac{z^{2} 2^{N} t^{2} e^{\lambda_{1}^{2} / 2}}{2}\right] \\
e^{-\phi}=\frac{1}{2 \sqrt{\pi}} \int_{-i \infty}^{i \infty} d z \exp \left(-z^{2} / 4+2^{N} e^{\left.-\frac{(\ln t+\ln z u)^{2}}{\lambda_{1}^{2}}\right)}\right.
\end{gathered}
$$

We obtain the following expressions for free energy. For the paramagnetic (PM) phase, without any magnetization, zeros of partition sum:

$$
\langle\ln |\operatorname{Re} Z|\rangle=N \ln 2+\frac{N\left(\beta_{1}^{2}-\beta_{2}^{2}\right)}{4}
$$

For the pin glass (SG) phase with some replica symmetry breaking:

$$
\langle\ln |\operatorname{Re} Z|\rangle=N \sqrt{\ln 2} \beta_{1}
$$


For the phase without any magnetization, but with some zeros for partition sum (further abbreviated as LYF; Lee-Yang [14, and Fisher [15]):

$$
\langle\ln |\operatorname{Re} Z|\rangle=\frac{1}{2} N \ln 2+\frac{\beta_{1}^{2}}{2} N
$$

Let us consider now the diluted version of the model. If we consider again our hamiltonian (1), only with \pm 1 couplings, then chose randomly among the all possible $C_{N}^{p}$ sets of indices $i_{1} . . i_{p}$ some $\alpha N$

$$
H=-\sum_{\left(i_{1}<i_{2} . .<i_{p}\right)=1}^{\alpha N} j_{i_{1} . . i_{p}} s_{i_{1}} . . s_{i_{p}}
$$

Now instead of the normal distributions we have

$$
\rho(E)=\frac{1}{2 \pi} \int_{-i \infty}^{i \infty} d E_{1} e^{-E E_{1}-\alpha N \ln c h E_{1}}
$$

Here $\alpha>1$ is a ratio of couplings number to spins number. For function $f(A, \lambda)$ we have

$$
\begin{gathered}
\frac{1}{2 \pi} \int_{-i \infty}^{i \infty} d E_{1} e^{\alpha N \ln \cosh \beta E_{1}} \int_{-\infty}^{\infty} d E e^{-\beta E E_{1}-\frac{i A}{2}\left(e^{\beta E}+e^{\bar{\beta} E}\right)}= \\
=\frac{1}{2 \pi} \int_{-i \infty}^{i \infty} d x e^{\alpha N \ln \cosh \beta x+x \ln A} \int_{0}^{\infty} e^{\frac{i}{2}\left(y^{1+\frac{i \beta_{2}}{\beta_{1}}}+y^{\left.1-\frac{i \beta_{2}}{\beta_{1}}\right)} y^{-x} d y=\right.} \\
=\frac{1}{2 \pi} \int_{-i \infty}^{i \infty} d x e^{\alpha N \ln \cosh \beta x-x \ln A} g(x, A)
\end{gathered}
$$

Again pole at point 0 gives

$$
f(A, \lambda) \approx 1-e^{\alpha N \ln \cosh \beta x-x \ln A}, \tanh \beta x=\frac{\ln A}{\alpha N \beta}
$$

This asymptotics is correct, when

$$
\tanh \beta>\frac{\ln A}{\alpha N \beta}
$$

To calculate another types of asymptotics we have to consider the derivatives $f_{A}^{\prime}, f_{A}^{\prime \prime}$.

$$
\begin{gathered}
f_{A}^{\prime}=\frac{i}{4 \pi} \int_{-i \infty}^{i \infty} d E e^{-E \hat{E}_{1} \beta+\alpha N \ln \cosh \beta E_{1}+\frac{i A}{2}\left(e^{\beta E}+e^{\bar{\beta} E}\right)}\left(e^{\beta E}+e^{\bar{\beta} E}\right)= \\
=\frac{i}{4 \pi} \int_{-\infty}^{\infty} d E e^{E \hat{E}_{1} \beta+\alpha N \ln \cosh \beta\left(E_{1}+1\right)+\frac{i A}{2}\left(e^{\beta E}+e^{\bar{\beta} E}\right)}+ \\
+\frac{i}{4 \pi} \int_{-i \infty}^{i \infty} d E e^{-E E_{1} \beta+\alpha N \ln \cosh \beta\left(E_{1}+\bar{\beta}_{1} / \beta\right)+\frac{i A}{2}\left(e^{\beta E}+e^{\bar{\beta} E}\right)}= \\
=\frac{i}{4 \pi} \int_{-i \infty}^{i \infty} e^{\alpha N \ln \cosh \beta(x+1)+x \ln A} g(x, A) d x+ \\
+\frac{i}{4 \pi} \int_{-i \infty}^{i \infty} e^{\alpha N \ln \cosh \beta(x+\bar{\beta} / \beta)+x \ln A} g(x, A) d x
\end{gathered}
$$


We have to move integration line left to catch the sadle point. Again we intersect the pole at point 0 .

$$
f(A, \lambda) \approx 1+\frac{i A}{2}\left(e^{\alpha N \ln \cosh \beta}+e^{\alpha N \ln \cosh \bar{\beta}}\right)
$$

This asymptotics is correct if for a solution

$$
\frac{\ln A}{\alpha N \beta}=\tanh (\bar{\beta}+\beta x)
$$

we have the constraint $\operatorname{Rex}<0$.

Let us consider now the third type of asymptotics. The consideration of second derivative reads:

$$
\begin{aligned}
\frac{d^{2} f}{d A^{2}}=-\frac{1}{8 \pi} \int_{-i \infty}^{i \infty} d E e^{-E E_{1} \beta+\alpha N \ln \cosh \beta E_{1}+\frac{i A}{2}\left(e^{\beta E}+e^{\bar{\beta} E}\right)}\left(e^{2 \beta E}+e^{2 \bar{\beta} E}+2 e^{2 \beta_{1} E}\right)= \\
=-\frac{1}{4 \pi} \int_{-i \infty}^{i \infty} e^{\alpha N \ln \cosh \left(x+2 \beta_{1}\right)-E_{1} \ln A} g(x) d x \\
-\frac{1}{8 \pi} \int_{-i \infty}^{i \infty} e^{\alpha N \ln \cosh (x+2 \beta)-E_{1} \ln A} g(x) d x \\
-\frac{1}{8 \pi} \int_{-i \infty}^{i \infty} e^{\alpha N \ln \cosh (x+2 \bar{\beta})-E_{1} \ln A} g(x)
\end{aligned}
$$

The shift of integration line intersects a pole, and gives

$$
\frac{d^{2} f}{d A^{2}}=-\frac{1}{8 \pi}\left[2 e^{\alpha N \ln \cosh \left(2 \beta_{1}\right)}-e^{\alpha N \ln \left(\beta E_{1}+2 \beta_{1}\right)-E_{1} \ln A}\right]
$$

where

$$
\begin{gathered}
\alpha N \beta \tanh \left(\beta E_{1}+2 \beta_{1}\right)=-\ln A \\
A-1 \approx-\frac{1}{4 \pi} e^{\alpha N \ln \cosh \left(2 \beta_{1}\right)}
\end{gathered}
$$

Again we have 3 mods.

$$
\begin{gathered}
e^{-\phi}=\frac{1}{2 \sqrt{\pi}} \int_{-i \infty}^{i \infty} e^{-z^{2} / 4+z i 2^{N} t\left[e^{\alpha N \ln \cosh (\beta)}+e^{\alpha N \ln \cos (\bar{\beta})}\right]} \\
e^{-\phi}=\frac{1}{2 \sqrt{\pi}} \int_{-i \infty}^{i \infty} \exp \left[-z^{2} / 4-2^{N} e^{-\frac{(\ln t+\ln z)^{2}}{\lambda_{1}^{2}}}\right] \\
e^{-\phi}=\frac{1}{2 \sqrt{\pi}} \int_{-i \infty}^{i \infty} \exp \left(-z^{2} / 4-z^{2} 2^{4} N t^{2} e^{\alpha N \ln \cosh 2 \beta_{1}}\right)
\end{gathered}
$$

Eventually we have three expressions for free energy. For the para magnetic phase:

$$
<\ln |\operatorname{Rez}|>=N \ln 2+\alpha N R e \ln \cosh \beta
$$

For the LYF phase:

$$
<\ln |\operatorname{Re} z|>=\frac{N}{2} \ln 2+\frac{\alpha N}{2} \ln \cosh 2 \beta_{1}
$$


For the SG phase:

$$
<\ln \operatorname{Re}|z|>=\alpha N \beta_{1} \tanh \beta_{c}
$$

where for the $\beta_{c}$ we have

$$
\alpha\left[\beta_{c} \tanh \beta_{c}-\ln \cosh \beta_{c}\right]=\ln 2
$$

The search of other possible phases like

$$
\frac{\alpha N}{4} \ln \cosh 4 \beta_{1}+\frac{N}{4} \ln 2
$$

gives the same equations, as for the case of real $T$. So we have not any new phase, besides those obtained in Eqs. (37-39). The boundary lines between 2 phases one can be found from the coincidence of 2 equations .

The boundary between LYF and SG phase is the line

$$
\beta_{1}=\beta_{0}, \beta_{0}<\beta_{2}<\infty
$$

where the multicriticity point $\left(\beta_{1}, \beta_{0}\right)$ lives on the intersection of line 44$)$ with the boundary SG, LYF.

$$
\sin ^{2} \beta_{2}=\frac{2^{1 / \alpha}-1-\tanh ^{2} \beta_{1}}{1-\tanh ^{2} \beta_{1}}
$$

For the case of ferromagnetic couplings we have the ferromagnetic phase with the old expression with $\beta_{1} \neq 0, \beta_{2}=0$. Let us consider the density of partition zeros near the $\beta_{1}$ axes. For the density of zeros we have [9] expresion

$$
\frac{1}{2 \pi}\left(\frac{d^{2}}{d \beta_{1}^{2}}+\frac{d^{2}}{d \beta_{1}^{2}}\right)<\ln |Z|>
$$

For the LY phase we have

$$
\rho\left(\beta_{1}, \beta_{2}\right)=\frac{1}{\pi} \frac{\alpha}{\cosh \left(\beta_{1}\right)^{2}}
$$

Near the PM-SG transition line we obtain:

$$
\rho\left(\beta_{1}, \beta_{2}\right)=\frac{1}{\pi} \frac{\alpha}{\cosh \left(\beta_{1}\right)^{2}} \delta\left(\beta_{1}+\beta_{2}-\beta_{c}\right)\left(\beta_{1}-\beta_{c}\right)
$$

Now we consider the phase structure of Generalized Random Energy Model in the domain of complex T. The SG phase transition conditions are the old ones, with the $\beta_{1}$ instead of $1 / T$. like

The conditions for phase transition to 3-rd (LYF) phase is more complicated. We have to consider an expression

$$
<\prod_{\alpha} \prod_{\beta_{\alpha}} \exp i \frac{A}{2}\left[e^{\lambda_{\alpha} E_{\alpha}+\lambda_{\beta} E_{\beta}}+e^{\bar{\lambda}_{\alpha} E_{\alpha}+\bar{\lambda}_{\beta} E_{\beta}}\right]>
$$

while expanding the exponent (to get LYF phase), we found

$$
<\prod_{\alpha} \prod_{\beta_{\alpha}} 1-A^{2} / 4\left[e^{\left(\lambda_{\alpha}+\bar{\lambda}_{\alpha}\right) E_{\alpha}+\left(\lambda_{\beta}+\bar{\lambda}_{\beta}\right) E_{\beta}}\right]>
$$

This expression showes, that if at some level of hierarchy exist LY phase, then higher could not exist PM, so stays only the SG one.

Let us consider the diluted version of GREM [13], with infinite number of hierarchy $M$. We can consider the case of large $M$ with smooth distribution of $z_{k}$ (number of couplings at level k) and $N_{k}$ (number of $\mathrm{k}$-th level branches is $2^{N_{k}}$ ). In this case we can introduce continuous variable $v=\frac{k}{M}$ between 0 and 1 , labeling the level of hierarchy and define distributions

$$
z_{k} \equiv \mathrm{d} z=z \mathrm{~d} v, \quad N_{k} \equiv \mathrm{d} N=n^{\prime}(v) \mathrm{d} v \quad \mathrm{~d} v=\frac{1}{M}
$$


where $n(v)$ is a given function (entropy in bits-s). The variable $v(0<v<1)$ parameterizes the level of the hierarchical tree and $z$ is just a parameter (for our spin system $\mathrm{z}$ is a total number of couplings and parameter $v$ labeling the level of hierarchy).

Similarly to the case of dilute GREM at real $\mathrm{T}$ we found:

$$
\begin{aligned}
-\frac{\beta F}{N}= & z\left(1-v_{2}(\beta)\right) R e \ln \cosh \beta+n\left(v_{2}(\beta)\right) \ln 2+n\left(v_{2}(\beta)\right) \ln 2 / 2 \\
& +z\left(v_{2}(\beta)-v_{1}(\beta)\right) / 2 \ln \cosh 2 \beta_{1}+z \beta_{1} \int_{0}^{v_{1}(\beta)} \mathrm{d} v_{0} g\left(\frac{z}{n^{\prime}\left(v_{0}\right)}\right)
\end{aligned}
$$

where $v_{2}(\beta), v_{1}(\beta)$ are defined from the equations

$$
\begin{aligned}
& 2 z R e \ln \cosh \beta+n^{\prime}\left(v_{2}(\beta)\right) \ln 2=z \ln \cosh 2 \beta_{1} \\
& z R e \ln \cosh \beta+n^{\prime}\left(v_{1}(\beta)\right) \ln 2=\beta_{1} z g\left(z / n^{\prime}\left(v_{1}\right)\right)
\end{aligned}
$$

and function $g(x)$ from the

$$
\left.\frac{1}{2}(1+g) \ln (1+g)+\frac{1}{2}(1-g) \ln (1-g)\right]=\frac{\ln 2}{x}
$$

For the case of Edwards-Anderson model placed on d-dimensional hypercubic lattice

$$
z=N d, \quad E=-v N d \quad n(v)=\frac{N s(-v d N)}{\ln 2}
$$

here $E$ is energy, and $s(E)$ is entropy as function of the energy for corresponding ferromagnetic Ising model. At given $\tilde{\beta}_{0}$ we can define corresponding $v_{0}$ as minus energy per bond for ferromagnetic model at temperature $\frac{1}{\tilde{\beta}_{0}}$.

$$
v_{0}\left(\tilde{\beta}_{0}\right)=-\frac{E\left(\tilde{\beta}_{0}\right)}{N d}
$$

We can remember from thee definition of temperature

$$
\frac{\mathrm{d} s}{\mathrm{~d} E}=\frac{1}{\tau} \equiv \tilde{\beta}
$$

We obtain for the free energy

$$
\begin{gathered}
-\frac{\beta F}{N d}=\left(1-v_{2}(\beta)\right) R e \ln \cosh \beta+s\left(v_{2}(\beta)\right)+\left(v_{2}(\beta)-v_{1}(\beta)\right) / 2 \ln \cosh 2 \beta_{1} \\
+s\left(v_{2}(\beta)\right) /(2 N d)+\beta_{1} \int_{0}^{v_{1}(\beta)} \mathrm{d} v_{0} f\left(\frac{\ln 2}{\tilde{\beta}\left(v_{0}\right)}\right)
\end{gathered}
$$

Integrating by parts in the last term we get

$$
\begin{gathered}
-\frac{\beta F}{N d}=\left(1-v_{2}(\beta)\right) R e \ln \cosh \beta+s\left(v_{2}(\beta)\right)\left(v_{2}(\beta)-v_{1}(\beta)\right) / 2 \ln \cosh 2 \beta_{1} \\
\quad+\left(s\left(v_{2}(\beta)-s\left(v_{1}(\beta)\right)\right) /(2 N d)-\beta_{1} \int_{0}^{\tilde{\beta}_{1}} \mathrm{~d} \tilde{\beta}_{0} \frac{2 v_{0}\left(\tilde{\beta}_{0}\right)}{\ln \frac{1+y}{1-y}}+v_{1}(\beta) \beta_{1} y\left(\tilde{\beta}_{1}\right)\right.
\end{gathered}
$$

where $y$ as a function of $\tilde{\beta}_{0}$ is defined from the equation

$$
y=g\left(\frac{\ln 2}{\tilde{\beta}_{0}}\right)
$$


function $v_{0}(\tilde{\beta})$ is defined from the $(54)$. and $\tilde{\beta}_{1}(\beta)$ is defined from the equation

$$
v_{1}(\beta)=-E\left(\tilde{\beta}_{1}\right) /(N d)
$$

where $v_{1}(\beta)$ is defined from the $(51)$. Here $E\left(\tilde{\beta}_{1}\right)$ is the energy of ferromagnetic model on the same lattice.

We propose the method for calculating approximate statistical mechanics of disordered models on finite lattices. It allows us also to identify Lee-Yang-Fisher zeroes. In the case of real temperatures our generalization of REM gave $5 \%$ accuracy. If such an accuracy will be available here, then this method could be very efficient.

We could succeed due to a simple observation (48) that on hierachy of generalized REM new (Lee-Yang-Fisher) phase could exist only on levels between paramagnetic and spin glass. On the other hand, there is no replica symmetry breaking. It is very interesting to consider the similar issues in strings where the ideas and methods of spin-glass theory have been applied recently.

We thank Fundacion Andes grant-C-13413/1 for a partial support.

[1] B. Derrida, Phys. Rev. Lett. 45 (1980) 79.

[2] J.P. Bouchaud, M. Mezard, J. Phys. 30A (1997) 7997.

[3] C.C. Chamon, C. Mudry, X.G. Wen, Phys. Rev.Lett. 77 (1996)4194.

[4] I.I. Kogan, C. Mudry, X.G. Wen, Phys. Rev.Lett. 77 (1996)707.

[5] H.E. Castillo et. al. , Phys. Rev. 56B (1997) 10668.

[6] N. Sourlas, Nature 239 (1989) 693.

[7] D. Saakian, cond-mat/9709238, JETP Lett.,1998,v.67,n.5

[8] C. Moukarzel, N. Parga, Physica A177 (1991) 24-30

[9] B. Derrida, Physica A177 (1991) 31-37

[10] B. Derrida, M.R. Evans, E.R. Speer, J. Stat. Phys., 156 (1993)

[11] D. Dominicis, P. Mottishow, J. Phys., A20 (1987)L267

[12] A.E. Allakhverdyan, D.B. Saakian, Nucl. Phys v.B498 (1997)604.

[13] D.B. Saakian, JETP Lett. v.55 (1992) 798.

[14] C.N. Yang and T.D. Lee, Phys. Rev. 87 (1952) 404; Phys. Rev. 87 (1952) 410.

[15] M.E. Fisher, in Lectures in Theoretical Physics, ed. by W.E. Brittin, (University of Colorado Press, Boulder, 1965), vol. $7 \mathrm{c}$.

[16] A.E. Allahverdyan, A.S. Ananikian, S.K. Dallakyan, Phys. Rev. E, 57 (1998) 2452.

[17] C. Itzykson, R.B. Pearson, and J.B. Zuber, Nucl. Phys. B, 220 (1983) 415.

[18] R.B. Griffiths, Phys. Rev. Lett., 23 (1969) 17. 